\title{
Spontaneous rupture of primary splenic angiosarcoma: A case report
}

\author{
BOTAO XU ${ }^{1,2}$, XIAONA XIE $^{3}$, XIONG ZHOU $^{1}$, MENGHE ZHAI $^{1}$ and WENJUN YANG ${ }^{1}$ \\ ${ }^{1}$ Division of General Surgery, First Affiliated Hospital of Wenzhou Medical University, Wenzhou, Zhejiang; \\ ${ }^{2}$ Department of General Surgery, Zhuji City People's Hospital of Zhejiang Province, Zhuji, Zhejiang; \\ ${ }^{3}$ Pulmonary Division, Wenzhou Traditional Chinese Medicine Hospital, Wenzhou, Zhejiang, P.R. China
}

Received October 18, 2014; Accepted July 30, 2015

DOI: $10.3892 / \mathrm{ol} .2015 .3714$

\begin{abstract}
Primary angiosarcoma of the spleen is an extremely rare malignant neoplasm of vascular origin that often has a poor prognosis. The majority of cases presents with splenic rupture and hemorrhage. The present study retrospectively analyzed the case of 77-year-old female who presented with diffuse abdominal pain and distension. During laparotomy, a huge actively bleeding spleen was identified and a splenectomy was performed. Since an accurate diagnosis could not be achieved by abdominal computed tomography, a pathological examination was performed. The patient and the family refused post-operative adjuvant chemotherapy and radiation therapy. The patient succumbed to uncontrolled hemorrhagic shock and lung metastases at 2 weeks post-surgery.
\end{abstract}

\section{Introduction}

Primary angiosarcoma of the spleen is an extremely rare, highly malignant neoplasm, the pathogenesis of which is unknown, with early systemic metastatic spread and a dismal prognosis, regardless of the treatment regimen (1). The disease is one of the least common types of cancer, with a reported incidence of $0.14-0.25$ cases per million individuals $(2,3)$. The majority of cases have median survival rates ranging between 4 and 18 months $(1,4)$. Splenic angiosarcoma was first described by Langhans in 1879 (5) and to date, 300 cases have been reported worldwide (6). Early metastasis is common and the most common sites include the liver, lungs, lymph nodes and gastrointestinal tract (7). The clinical presentation of splenic angiosarcoma is usually unspecific; symptoms of abdominal pain and anaemia are commonly reported (8). Forming a diagnosis may be difficult due to the atypical presentation of the

Correspondence to: Dr Wenjun Yang, Division of General Surgery, First Affiliated Hospital of Wenzhou Medical University, South Baixiang Street, Wenzhou, Zhejiang 325000, P.R. China E-mail: yangwenjun165@sohu.com

Key words: primary splenic angiosarcoma, splenic rupture, clinical characteristics, radiographic features tumor. A diagnosis of splenic angiosarcoma should be considered in patients with splenomegaly and anemia of unknown etiology (9). Primary splenic angiosarcoma is usually treated surgically and splenectomy is the mainstay of treatment, as the lesion is highly refractory to adjuvant treatment with radiation and chemotherapy (10). Emergency splenectomy is the standard treatment for cases exhibiting ruptured splenic angiosarcoma (8). Splenic rupture is a serious complication of the disease, which is frequently observed in patients, that results in mortality in a significant proportion of cases (7). The prognosis of primary angiosarcoma of the spleen remains poor as liver, lung and bone metastases are common (8). The current study presents a case of spontaneous splenic rupture in a 77-year-old female who was treated by laparotomy and splenectomy. Written informed consent was obtained from the patient's family.

\section{Case report}

In June 2014, a 77-year-old female was admitted to the Department of General Surgery, First Affiliated Hospital of Wenzhou Medical University (Wenzhou, Zhejiang, China) with diffuse abdominal pain and distension that had been apparent for 6 days. A physical examination revealed abdominal distension and a palpable abdominal mass in the left upper abdominal quadrant. An initial laboratory test revealed thrombocytopenia (platelet count, 7.0x10 $10 / 1$ ) and anemia (hemoglobin level, $7.0 \mathrm{~g} / \mathrm{dl}$ ). Results of other laboratory examinations were not reported prior to surgery. Abdominal ultrasonography showed a large amount of intraperitoneal free fluid and an enlarged spleen filled with irregular nodules. These findings were confirmed by an abdominal computed tomography (CT) scan (Fig. 1). CT revealed a large intraperitoneal hematoma in the left upper quadrant.

Laparotomy revealed a huge, actively bleeding spleen and an abnormal liver, with several metastatic foci. Blood (2 1) filled the intraperitoneum. A crack was observed in the spleen, measuring $4 \times 4 \mathrm{~cm}$ in depth, in the upper pole. A splenectomy was performed. During the surgical procedure, the patient received 2 units of concentrated red blood cells and 2 units of fresh frozen plasma.

The spleen was $1,550 \mathrm{~g}$ in weight and 19x16x11 cm in size, with a nodular appearance and bleeding. The pathological examination confirmed that the excised spleen was 


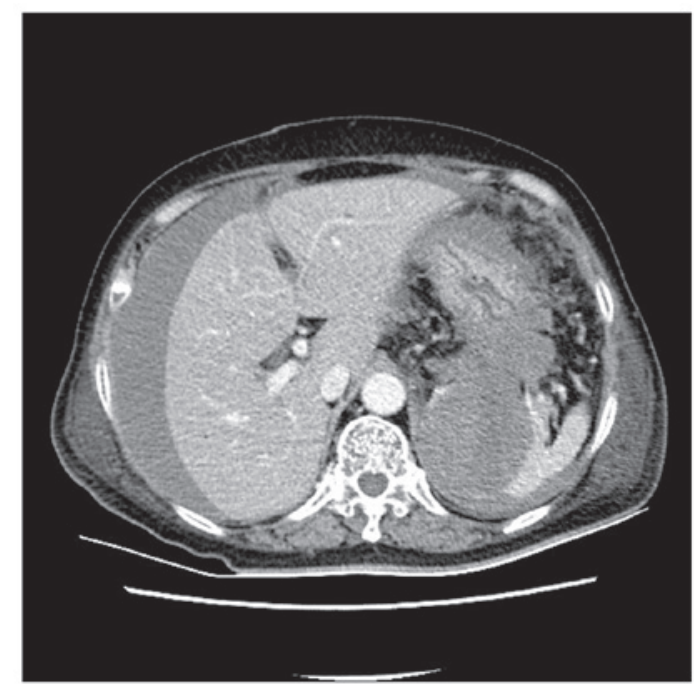

Figure 1. Contrast-enhanced image in the portal venous phase computed tomography scan showing the enlarged spleen completely replaced by tumor tissue with low density. Free fluid is also visible around the liver and spleen, indicating intraperitoneal hemorrhage.

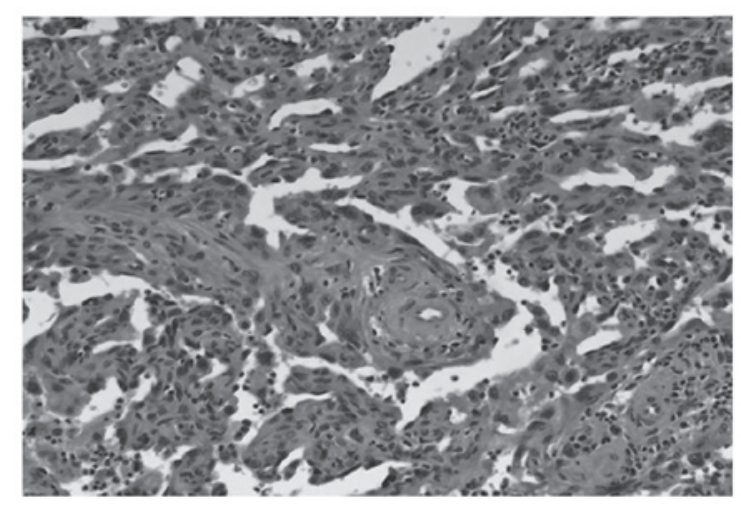

Figure 2. Histopathological findings of angiosarcoma of the spleen. Spindle tumor cells have replaced the normal pulp in the spleen, while ecstatic vascular spaces lined with hypertrophied endothelial cells are apparent (stain, hematoxylin and eosin; magnification, x200).

an angiosarcoma, presumably of splenic origin (Fig. 2); the spleen was composed of spindle tumor cells and the ecstatic vascular spaces were lined with hypertrophied endothelial cells. Immunohistochemical examination demonstrated positive immunostaining for cluster of differentiation (CD)31 and CD34.

The patient refused any further treatment and two weeks after surgery, the patient succumbed to the disease due to hemorrhagic shock and lung metastases.

\section{Discussion}

Primary angiosarcoma of the spleen is an uncommon and aggressive malignant neoplasm that is derived from the splenic vascular endothelium and elongated endothelial cells of mesenchymal origin that line the spongy network of sinusoids within the spleen (9). In general, angiosarcomas are rapid proliferating, highly infiltrating anaplastic tumors that tend to recur locally, be widely disseminated, and have an increased rate of lymph node and systemic metastases $(11,12)$. The primary histological feature of an angiosarcoma is the formation of vascular channels with a sarcomatous stroma and papillary appearance due to endothelial cell proliferation, although another common finding is an undifferentiated neoplasm (7).

The etiology of primary splenic angiosarcoma remains unknown. Causes of this disease have been reported as exposure to ionizing radiation or chemotherapy for lymphoma. Besides that, exposure to certain chemical agents, such as vinyl chloride, thorium dioxide and arsenic have been indicated due to their association with hepatic angiosarcomas (13).

The clinical manifestations of primary splenic angiosarcoma are rather variable, including abdominal pain, splenomegaly, anemia, fatigue, generalized weakness, fever, weight loss and even life-threatening hemorrhage resulting from rupture of the spleen. Left upper abdominal pain is the most common symptom. Constitutional symptoms common in malignancy, such as fever, fatigue and weight loss, have also been observed but are the initial symptoms $<10 \%$ of the time. The most common physical examination is splenomegaly. Splenic rupture is the most serious manifestation, which often leads to fatal hemorrhage (7).

Laboratory tests can reveal pancytopenia, leukocytosis, thrombocytosis and an elevated erythrocyte sedimentation rate prior to diagnosis (7). Anemia is the most common laboratory abnormality, being found in $75-81 \%$ of cases, although $10-40 \%$ of patients exhibit thrombocytopenia; leukocytosis is also often noted (9). One of the serious complications that is frequently observed in patients with splenic angiosarcoma is splenic rupture, leading to a fatal outcome in a significant percentage of cases $(6,7)$.

Imaging modalities are invaluable for the differential diagnosis from other benign and malignant splenic tumors, although diagnostic accuracy is lacking. CT scans may reveal splenic enlargement with hypo- or hyper-attenuating areas (7). On contrast-enhanced CT scans, the tumors may show peripheral or heterogeneous contrast enhancement similar to that of hepatic cavernous hemangiomas. However, the clinical features and radiological appearance of the majority of cases are quite varied and non-specific, and may be easily associated with other pathological conditions, which makes early diagnosis and treatment difficult (6).

The usual therapy for ruptured splenic angiosarcoma is an emergency splenectomy. At present, there is no convincing evidence to suggest a clinical benefit of chemotherapy in the treatment of splenic angiosarcoma. When considering early hematogenous micrometastasis, systemic chemotherapy following surgery may be theoretically beneficial (6). Although there is no standard treatment regimen, certain drugs, including cyclophosphamide, doxorubicin, epirubicin, ifosfamide, daunorubicin, vincristine, actinomycin D, steroids and Taxol, which have shown efficacy for treating angiosarcoma in other anatomical sites or other types of soft-tissue sarcomas, have been empirically proposed to be effective for primary splenic angiosarcoma (14).

The prognosis of patients with primary splenic angiosarcoma is quite poor. The majority of patients succumb to systemic metastases within 1-2 years of diagnosis, even when 
the primary tumor has been removed (15). Long-term survival has only previously been reported in patients who presented without metastases $(6,16)$. However, certain studies have considered splenic rupture to be the worst prognostic factor, as this places patients at a high risk of peritoneal or vascular dissemination (7). This finding is confirmed by the fact that early splenectomy prior to rupture of the organ is accompanied by better survival rates (6).

In conclusion, primary splenic angiosarcoma is an extremely rare and aggressive soft-tissue sarcoma due to the presence of early systemic metastases and life-threatening hemorrhage from the rupture of the spleen. The pathogenesis of the disease remains unclear, and the clinical and radiological diagnoses are challenging. The diagnosis of primary splenic angiosarcoma should be suspected in any patient with splenomegaly and anemia of unknown etiology. Radiation and chemotherapy have historically been unsuccessful in improving outcomes in this patient population. Further evaluation of these options will be required due to the limited experience to date. The best chance for survival follows an early diagnosis and a prompt splenectomy prior to splenic rupture.

\section{References}

1. Duan YF, Jiang Y, Wu CX and Zhu F: Spontaneous rupture of primary splenic angiosarcoma: A case report and literature review. World J Surg Oncol 11: 53, 2013.

2. Falk S, Krishnan J and Meis JM: Primary angiosarcoma of the spleen. A clinicopathologic study of 40 cases. Am J Surg Pathol 17: 959-970, 1993.

3. Neuhauser TS, Derringer GA, Thompson LD, Fanburg-Smith JC, Miettinen M, Saaristo A and Abbondanzo SL: Splenic angiosarcoma: A clinicopathologic and immunophenotypic study of 28 cases. Mod Pathol 13: 978-987, 2000.
4. Buckner JW III, Porterfield G and Williams GR: Spontaneous splenic rupture secondary to angiosarcoma. J Okla State Med Assoc 83: 211-213, 1990.

5. Langhans T: Pulsating cavernous neoplasm of the spleen with metastatic nodules to the liver. Vichows Arch Pathol Anat 75: 273-291, 1879.

6. Liu Z, Du X, Li H, Wang Z, Shen Z, Yao Y and Zhao H: Primary splenic angiosarcoma. Vasa 41: 57-62, 2012.

7. Manouras A, Giannopoulos P, Toufektzian L, Markogiannakis H, Lagoudianakis EE, Papadima A, Papanikolaou D, Filis K and Kekis P: Splenic rupture as the presenting manifestation of primary splenic angiosarcoma in a teenage woman: A case report. J Med Case Rep 2: 133, 2008.

8. Alexandrino H, Julião MJ, Tralhão JG and Sousa FC: Rupture of splenic angiosarcoma: A rare cause of spontaneous haemoperitoneum. BMJ Case Rep 24: 2013, 2013.

9. Kamran S, Rodriguez JA and Lairmore TC: Primary splenic angiosarcoma. JSLS 14: 431-435, 2010.

10. Despoina M, Dionysios D, Georgios A, Konstantinos S, Efstratios K and Adamantia ZS: Primary angiosarcoma of the spleen: An oncological enigma. Case Rep Oncol Med 2014: 193036, 2014.

11. Abbott RM, Levy AD, Aguilera NS, Gorospe L and Thompson WM: From the archives of the AFIP: Primary vascular neoplasms of the spleen: Radiologic-pathologic correlation. Radiographics 24: 1137-1163, 2004.

12. Thompson WM, Levy AD, Aguilera NS, Gorospe L and Abbott RM: Angiosarcoma of the spleen: Imaging characteristics in 12 patients. Radiology 235: 106-115, 2005.

13. den Hoed ID, Granzen B, Granzen B, Aronson DC, Pauwels P, de Kraker J and van Heurn LW: Metastasized angiosarcoma of the spleen in a 2-year-old girl. Pediatr Hematol Oncol 22: 387-390, 2005

14. Ferreira BP, Rodler ET, Loggers ET, Pollack SM and Jones RL: Systemic therapy in primary angiosarcoma of the spleen. Rare Tumors 4: e55, 2012.

15. Kren L, Kaur P, Goncharuk VN, Dolezel Z and Krenova Z: Primary angiosarcoma of the spleen in a child. Med Pediatr Oncol 40: 411-412, 2003.

16. Hsu JT, Ueng SH, Hwang TL, Chen HM, Jan YY and Chen MF: Primary angiosarcoma of the spleen in a child with long-term survival. Pediatr Surg Int 23: 807-810, 2007. 